\title{
Astrocytes in Culture Synthesize and Secrete a Variant Form of Fibronectin ${ }^{1}$
}

\author{
JACK PRICE AND RICHARD O. HYNES \\ Center for Cancer Research, Massachusetts Institute of Technology, Cambridge, Massachusetts 02139
}

\begin{abstract}
Astrocytes in culture have very little cell surface fibronectin as detected by iodination or immunocytochemistry. Nonetheless, they synthesize and secrete this glycoprotein in amounts comparable with the production by fibroblasts. Astrocyte fibronectin has properties in common with other forms of the protein. It binds to gelatin- and heparin-coupled Sepharose and it is recognized by specific anti-fibronectin sera. It also exists as a dimer under non-reducing conditions. However, by sodium dodecyl sulfate-polyacrylamide gel electrophoresis (SDS-PAGE), astrocyte fibronectin appears larger, under both reduced and non-reduced conditions, than other cellular fibronectins. This apparent size difference is not the result of post-translational modifications. If the cultures are treated with tunicamycin, the astrocytes produce fibronectin that is unglycosylated, as shown by $\left[{ }^{3} \mathrm{H}\right]$ glucosamine labeling, and is neither sulfated nor phosphorylated as indicated by $\left[{ }^{35} \mathrm{~S} \mathrm{O}_{4}\right.$ and $\left[{ }^{32} \mathrm{P}\right] \mathrm{O}_{4}$ labeling studies. This astrocyte-derived, carbohydrate-free fibronectin resolves on SDSPAGE as four bands, of which the heavier ones predominate. Fibroblasts produce a similar set of four bands, but in this form of fibronectin the less heavy bands predominate. Thus, we conclude that fibronectin is a major secreted protein of astrocytes in vitro and that these cells produce a variant form of the protein which is enriched in the higher molecular weight subunits.
\end{abstract}

Fibronectins (FNs) are a group of large glycoproteins which are found in basement membranes (BMs), extracellular matrices, and a number of body fluids, including plasma and cerebrospinal fluid (Hynes and Yamada, 1982; Furcht, 1983; Yamada, 1983). All of the proteins in the group are disulfide-bonded dimers with subunits of about 220,000 to 250,000 daltons, and all have a number of properties in common, such as heparin and golatin binding. They exist in many somatic tissues where they are thought to be important in such biological phenomena as cell adhesion and migration.

Received September 14, 1984; Revised December 10, 1984; Accepted December 11, 1984

\footnotetext{
This work was started and largely performed in Martin Raff's laboratory. We would like to thank Martin and all of our colleagues in the Medical Research Council Neuroimmunology Project, Department of Zoology, University College London for their hospitality, enthusiasm, generous help, and advice. We also thank Jeremy Paul for sharing his expertise in electrophoresis and for kind gifts of Nil 8 and Rat 1 labeled culture supernatants. We acknowledge the financial support of the United States Public Health Service National Cancer Institute (Grant PO1CA26712) and the Medical Research Council. R. O. H. was a John Simon Guggenheim Fellow. J. P. is the recipient of a European Molecular Biology Organization long-term fellowship.
}

Interestingly, these ubiquitous proteins are not detectable in most parts of the central nervous system (CNS), being found only in the BMs surrounding blood vessels and underlying the pia mater (Schachner et al., 1978; Paetau et al., 1980a; Minier et al., 1981; Jones et al., 1982). Where this CNS FN is synthesized is unknown, but it seems that astrocytes in culture synthesize laminin, another BM glycoprotein (Liesi et al., 1983), and astrocytes in vivo are often found to have their end-feet on the BMs of blood vessels and pia. Thus, astrocytes could be contributing both laminin and $F N$ to these BMs.

When astrocytes have been studied in culture, most workers have been unable to detect FN on the surfaces of these cells (Raff et al., 1979; Kennedy et al., 1980; Paetau et al., 1980b). Noncthcless, there have been some reports of astrocytes with surface FN as detected by immunocytochemistry (Vaheri et al., 1976; Rajaraman, 1978), although these workers did not employ markers-such as glial fibrillary acidic protein (GFAP) - to identify the $\mathrm{FN}^{+}$cells as astrocytes. In addition, many $\mathrm{GFAP}^{+}$gliomas do not have surface $\mathrm{FN}$, but there are a number that apparently do (Jones et al., 1982). One problem with studying cells by such immunocytochemical methods, however, is that one cannot distinguish between cells that are synthesizing $\mathrm{FN}$ and those which may merely be binding it or taking it up. This approach says nothing about the source of the bound FN. Neither does it indicate cells which might be secreting the protein but not binding it.

In this study, we have asked whether cultured astrocytes synthesize $\mathrm{FN}$, and whether they secrete it or bind it to their surfaces. We have addressed this question using metabolic labeling as well as immunocytochemistry. We show that astrocytes synthesize and secrete considerable amounts of FN in culture, but very little if any of this glycoprotein is incorporated into extracellular matrix. We also demonstrate that this astrocyte $\mathrm{FN}$ is distinguishable from other forms of the protein. The astrocyte form is of slightly higher molecular weight than the cellular form produced by fibroblasts, and we show this difference to be due to a preponderance of larger protein subunits rather than to any of the post-translational modifications found in FN (Paul and Hynes, 1984).

\section{Materials and Methods}

Cell culture. Cortical astrocytes were prepared by the modification of the procedure of McCarthy and DeVellis (1980) used by Rougon et al. (1983). Briefly, the cerebral cortices from 2-or 3-day-old rat pups were dissected away from other brain tissue and meninges, and chopped with fine scissors. The tissue was treated for 30 min with $0.025 \%$ trypsin $+0.02 \%$ EDTA and then dissociated by passage through a 21 gauge needle. After passage through sterile $140-\mu \mathrm{m}$ nylon gauze, the cells were plated onto $75-\mathrm{cm}^{2}$ tissue culture flasks (Falcon) coated with poly-L-lysine (PLL) at a density of $2 \times 10^{7}$ cells/flask. The cells were grown in Dulbecco's modified Eagle's medium (DMEM) $+10 \%$ fetal calf serum (FCS), with medium changes every 3 to 4 days. After about 9 days, a mixed culture had arisen which had a population of process-bearing cells (neurons, astrocytes, and oligodendrocytes) growing on a monolayer of flat fibroblastic cells (astrocytes, leptomeningeal cells, and fibroblasts). The process-bearing cells were removed by shaking overnight 
on a rotary shaker at 180 to $200 \mathrm{rpm}$ and then replacing the medium with fresh medium. The remaining flat cells were then trypsinized from the flask and transferred into five flasks. Twenty-four hours later, arabinosylcytosine $\left(10^{-5} \mathrm{M}\right)$ was added to the cultures for 2 more days. This kills dividing cells in the culture, and the monolayer that remains is made up of almost pure astrocytes (type 1 astrocytes according to the nomenclature of Raft et al., 1983), as defined by GFAP staining (see "Results"). For most experiments, the astrocytes were transferred one more time (giving tertiary cultures) onto 10- or 6-cm tissue culture dishes or Linbro multiwell plates and were used at corrfluency

Cultures of rat skin fibroblasts were produced by dissecting and chopping up the skin from 3-day-old rats and incubating it for $1 \mathrm{hr}$ with $0.05 \%$ collagenase dissolved in the digestion butter of Leffert et al. (19/9). The supernatant from the collagenase dissociation was passed through a 21 gauge needle, then through nylon gauze, and was plated onto $75-\mathrm{cm}^{2}$ flasks at a density of $10^{7}$ cells/flask. The cells were grown in DMEM $+10 \%$ FCS and fed every 3 to 4 days. When confluent, the cells were transferred at lower density to fresh flasks. Finally, tertiary cultures were grown on tissue culture plates and used for experimental procedures when confluent.

Schwann cells were produced from 1- to 2-day-old rats according to the method of Brockes et al. (1977)

Lactoperoxidase-mediated surface labeling. Cell surface proteins were labeled by lactoperoxidase-catalyzed iodination as described previously (Hynes, 1973). Confluent cultures of cells in 60-mm dishes were labeled for $10 \mathrm{~min}$ at room temperature in phosphate-buffered saline (PBS) containing 5 $\mathrm{mm}$ glucose, $400 \mu \mathrm{Ci} / \mathrm{ml}$ of carrier-free ${ }^{125}$-labeled iodide (Amersham International), $20 \mu \mathrm{g} / \mathrm{ml}$ of lactoperoxidase (Calbiochem-Behring), and $1 \mathrm{unit} / \mathrm{ml}$ of glucose oxidase (Worthington). After rinsing to remove excess iodide, cells either were lysed directly in electophoresis sample buffer (Laemmli. 1970) or were scraped from the dishes with a rubber policeman, centrifuged through a cushion of electrophoresis sample buffer lacking sodium dodecyl sulfate and dithiothreitol, and the pellet was dissolved in complete sample buffer. These samples were then run on sodium dodecyl sulfate-polyacrylamide gel electrophoresis (SDS-PAGE) according to the method of Laemmli (1970). Gels were prepared for fluorugraphy by treating them with Enhance (New England Nuclear) and then drying them down and exposing them to Kodak X-Omat AR film at $-70^{\circ} \mathrm{C}$ (Bonner and Laskey, 1974). Gels that were labeled with ${ }^{32} \mathrm{P}$ (see below) were not treated with Enhance.

Metabolic labeling. Four different types of labeling experiments were undertaken, all on parallel cultures: $\left[{ }^{35} \mathrm{~S}\right]$ methionine, $\left.{ }^{3} \mathrm{H}\right]$ glucosamine, $\left.{ }^{35} \mathrm{~S}\right]$ $\mathrm{O}_{4}$ and ${ }^{32} \mathrm{PJO}_{4}$. In each case, cells were transferred into DMEM containing $10 \%$ FCS from which FN had been removed by passage over gelatinSepharose (Engvall and Ruoslahti, 1977). Cultures that were to be labeled with $\left[{ }^{35} \mathrm{~S}\right]$ methionine or $\left[{ }^{3} \mathrm{H}\right]$ glucosamine received DMEM containing $0.1 \mathrm{~N}$ methionine or glucose as appropriate; cultures for $\left[{ }^{35} \mathrm{~S}^{\mathrm{O}} \mathrm{O}_{4}\right.$ labeling received sulfate-free DMEM + 10\% FN-depleted FCS which had also been dialyzed extensively against PBS to remove sulfate; and cultures to be labeled with ${ }^{32} \mathrm{P}_{3} \mathrm{O}_{4}$ were given phosphate-free DMEM $+10 \%$ FCS that had been extensively dialyzed against a $150 \mathrm{~mm}$ saline solution. Together with the appropriate fresh medium, cells received either $100 \mu \mathrm{Ci} / \mathrm{ml}$ of $\left[{ }^{3} \mathrm{H}\right] \mathrm{glucosa}$ mine, $50 \mu \mathrm{Ci} / \mathrm{ml}$ of $\left.{ }^{35} \mathrm{~S}\right]$ methionine, $200 \mu \mathrm{Ci} / \mathrm{ml}$ of $\left[{ }^{35} \mathrm{~S}_{\mathrm{O}} \mathrm{O}_{4}\right.$, or $500 \mu \mathrm{Ci} / \mathrm{ml}$ of $\left[{ }^{32} \mathrm{P}_{\mathrm{O}}\right.$. The cultures were incubated for $24 \mathrm{hr}$ at $37^{\circ} \mathrm{C}$; then, the medium was removed and phenylmethylsulfonyl fluoride (PMSF) and EDTA (both 2 $\mathrm{mm}$ ) were added. The supernatant was then spun at 10,000 $\times \mathrm{g}$ and stored in aliquots at $-20^{\circ} \mathrm{C}$

Some parallel cultures were treated $1 \mathrm{hr}$ before labeling with tunicamycin (TM) (Calbiochem) at 3 to $10 \mu \mathrm{g} / \mathrm{ml}$. These cultures were then labeled as above in the presence of TM. This concentration range for TM was chosen empirically as the lowest dose which brought about an almost total inhibition of $\left[{ }^{3} \mathrm{H}\right]$ glucosannire labeling into FN while inthibiling $\left[{ }^{35}\right.$ S $]$ rnelt hionine incorporation by the smallest amount possible-typically no more than $50 \%$ (see Fig. 5).

Gelatin- and heparin-coupled Sepharose binding. Aliquots of $100 \mu \mathrm{l}$ of gelatin-coupled Sepharose (made according to the method of Engvall and Ruoslahti, 1977), heparin coupled Sepharose (Pharmacia), or uncoupled Sepharose were preadsorbed with an equal volume of DMEM containing $10 \%$ FN-free FCS and $0.1 \%$ sodium azide. Each of these was then incubated for $3 \mathrm{hr}$ at room temperature with an equal volume of prespun, $\left[{ }^{35} \mathrm{~S}\right]$ methionine-labeled astrocyte culture supernatant, with occasional vortexing. These incubation mixtures were then centrifuged, and the supernatants were removed. The pelieted Sepharose was then washed three times with PBS containing $2 \mathrm{mM}$ PMSF and $2 \mathrm{mM}$ EDTA to remove any unbound material and was boled in electrophoresis sample buffer to solubilize the bound material. These samples were then analyzed by SDS-PAGE.

Immunoprecipitation. The labeled culture supernatants were immunopre- cipitated by incubating them with the rabbit anti-hamster FN described by Mautner and Hynes (1977) at a concentration of $20 \mu \mathrm{l}$ of antiserum $/ \mathrm{ml}$ of supernatant in the presence of $0.5 \%$ Nonidet P- 40 (NP-40) $+0.5 \%$ deoxycholate (DOC) $+0.1 \% \mathrm{SDS}+2.5 \mathrm{mg} / \mathrm{ml}$ of ovalbumin. After incubation for $1 \mathrm{hr}$ at $37^{\circ} \mathrm{C}$, the antigen-antibody complexes were precipitated by adding goat anti-rabbit lgG (Cappel Laboratories) at a concentration of 10 times that of the primary antiserum. The supernatant was incubated for $1 \mathrm{hr}$ at $37^{\circ} \mathrm{C}$ and overnight at $4^{\circ} \mathrm{C}$, and then was centrifuged; the pellet was washed three times in a wash buffer of Tris-HCl $(100 \mathrm{~mm}), \mathrm{pH} 8.1$, containing $0.1 \%$ SDS, $0.5 \% \mathrm{NP}-40,0.5 \%$ DOC, $2 \mathrm{~mm}$ CDTA, and $2 \mathrm{mM} \mathrm{PMST}$. As a control, some aliquots of supernatant received a similar volume of preimmune serum in place of the primary antiserum.

Immunocytochemistry. The purity of the astrocytes used for metabolic labeling experiments was monitored by staining for GFAP. This intermediate filament protein is found only in astrocytes in the CNS and has been widely used in culture as a definitive marker for this cell type. GFAP staining was done on cells which, at the time of the plating of the tertiary astrocytes used for metabolic labeling, were plated onto PLL-coated glass coverslips and grown at the same cell density and the same conditions as the cultures used for metabolic labeling. These coverslip cultures were fixed on the coverslip by $95 \%$ ethanol $/ 5 \%$ acetic acid for 10 to $15 \mathrm{~min}$ at $-20^{\circ} \mathrm{C}$. After a brief wash, the cells were incubated with rabbit anti-GFAP (Pruss, 1979), diluted $1: 200$ in PBS containing 5\% FCS, for $1 \mathrm{hr}$. After another brief wash, the coverslips were incubated with rhodamine-conjugated goat anti-rabbit immunoglobulin (Cappel) diluted 1:100 in the same dilution buffer as above. After a final brief wash, the coverslips were mounted in glycerol and viewed with a Zeiss epifluorescent microscope. In control experiments, the primary antiserum was replaced with a similar dilution of normal rabbit serum. As a separate control, fibroblasts grown on coverslips were stained with antiGFAP instead of astrocytes. Also, some fibroblast cultures were stained with anti-vimentin (Hynes and Destree, 1978).

In addition to the GFAP staining, coverslip cultures of both astrocytes and fibroblasts were stained with rabbit anti-FN antiserum. The method used was similar to the above except that (1) rather than prefixing with the acid alcohol, the cells were stained unfixed and fixed just prior to mounting, and (2) the FCS used in the antibody diluent was FN-free FCS.

\section{Results}

The immunocytochemical staining of the tertiary astrocyte cultures showed that 95 to $98 \%$ of these cells were $\mathrm{GFAP}^{+}$(Fig. 1). This confirms the results of McCarthy and DeVellis (1980) and of Rougon et al. (1983), who showed that almost pure astrocyte cultures could be produced by this procedure. All of the cells in the skin fibroblast cultures were $\mathrm{VIM}^{+}, \mathrm{FN}^{+}, \mathrm{GFAP}^{-}$.

Astrocyte cell surface FN. Two techniques were employed to assess whether. FN was present on the surface of cultured astrocytes: surface iodination and immunocytochemistry. The result of the iodination study is shown in Figure 2, where astrocytes are compared with fibroblasts and Schwann cells. Whereas fibroblasts have a strong characteristic FN band at 230,000 daltons, neither the astrocytes nor the Schwann cells have significant labeling in that region of the gel.

In the immunocytochemical study with anti-FN on living astrocytes, the results were largely negative. In no case did astrocytes have the extensive fibrillar FN matrix characteristic of fibroblasts. However, in some cases astrocytes did show some FN staining. Mostly it was low in amount and punctate rather than extensively fibrillar, but occasionally, fine fibrillar staining could be seen (Fig. 1b). Thus, by immunocytochemistry, anti-FN staining on astrocytes was either low or absent.

${ }^{35}$ SJMethionine labeling studies. Figure 3 shows SDS-PAGE analysis under reducing and non-reducing conditions of the culture supernatant from $\left[{ }^{35} \mathrm{~S}\right]$ methionine-labeled astrocytes and fibroblasts. One can see that, as expected, the fibroblasts have a very clear band rurning in the pusilion of FN dimer (non-reduced) or FN monomer (reduced). It is also clear that the astrocytes have a similar strong band. However, the astrocyte and fibroblast bands do not exactly co-migrate; the fibroblast FN band runs slightly ahead of the astrocyte band. The other bands detected in the culture medium probably include procollagens, based on their molecular weights and disulfide bonding. We have not attempted to identify them 

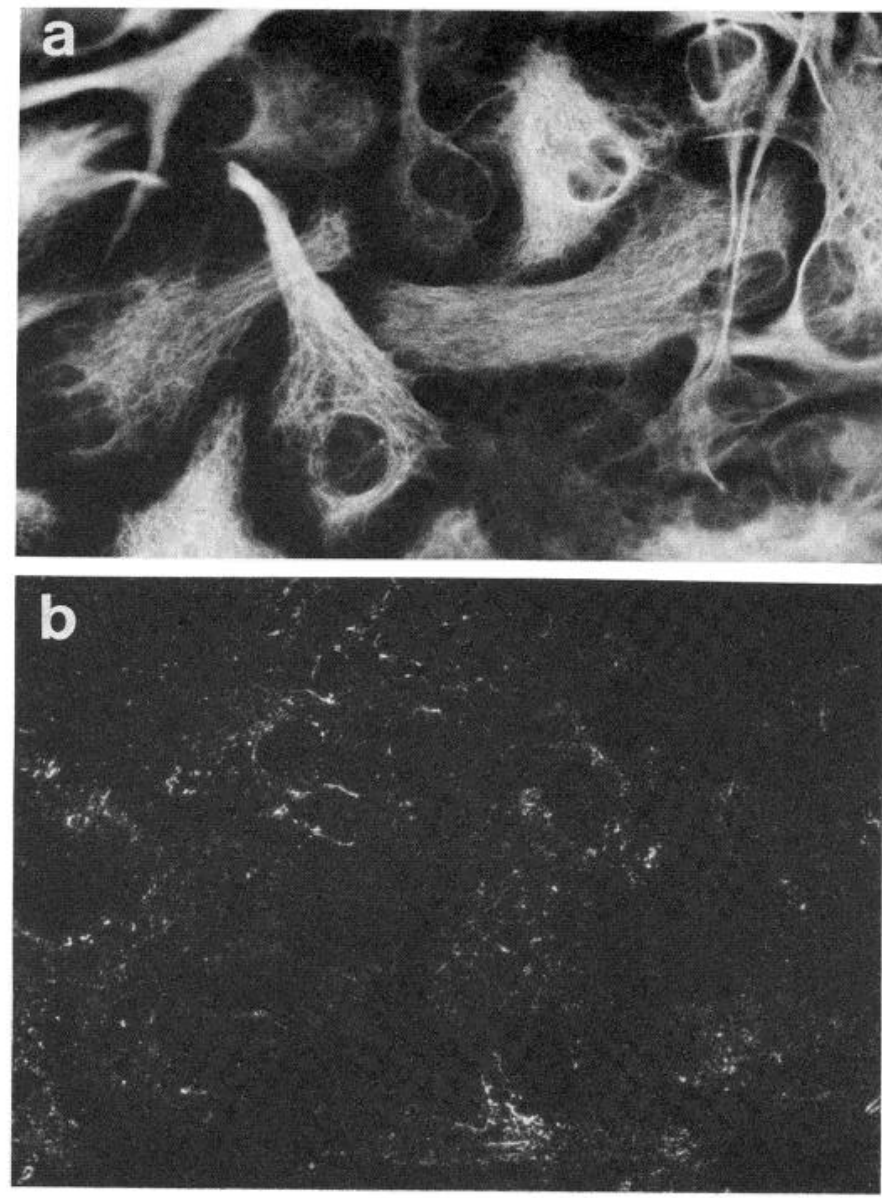

Figure 1. Astrocyte immunocytochemistry. Photomicrographs of cultured astrocytes stained with anti-GFAP $(a)$ and anti-FN $(b)$. Between 95 and $98 \%$ of the cells are $\mathrm{GFAP}^{+}$, but most show only a small amount of punctate or finely fibrillar FN staining. The photomicrographs are of sister cultures taken at the same magnification.

further. In parallel experiments with cultures of Schwann cells, we could detect only low levels of secreted FN which could have been due to contaminating fibroblasts (data not shown).

Gelatin and heparin binding studies. The $\left[{ }^{35} \mathrm{~S}\right]$ methionine labeling experiment showed that astrocytes produced large amounts of a protein that runs like FN on SDS-PAGE but is of a slightly higher apparent molecular weight than the fibroblast form of the protein. Although it seemed highly likely that this band was FN, more definitive criteria were required. Hence, we sought to show that this protein bound to gelatin and heparin, properties characteristic of FN. Figure 4 shows the result of passing the $\left[{ }^{35} \mathrm{~S}\right]$ methionine-labeled culture supernatant from astrocytes over gelatin- or heparin-coupled Sepharose. The original supernatant is shown for comparison together with the eluted samples. One can see that both heparin- and gelatin-coupled Sepharose bind the putative FN band from the astrocyte supernatant.

Immunoprecipitation. As further proof of the identity of the putative astrocyte $\mathrm{FN}$, we investigated whether the astrocyte protein was recognized by anti-FN antibodies. Figure $5 \mathrm{a}$ shows the result of immunoprecipitation of $\left[{ }^{35} \mathrm{~S}\right]$ methionine-labeled culture supernatants from fibroblasts and astrocytes. The figure shows the resulting precipitates run on SDS-PAGE under reducing conditions. Lanes 1 and 4 show the proteins precipitated from astrocyte and fibroblast supernatants, respectively. Lane 5 shows the preimmune serum control. Anti-FN serum specifically precipitates one broad FN band from both fibroblasts and astrocytes. In addition, there is a fainter band that runs slightly ahead of the major band. This arrangement of a major broad band plus a fainter, lighter one is characteristic of
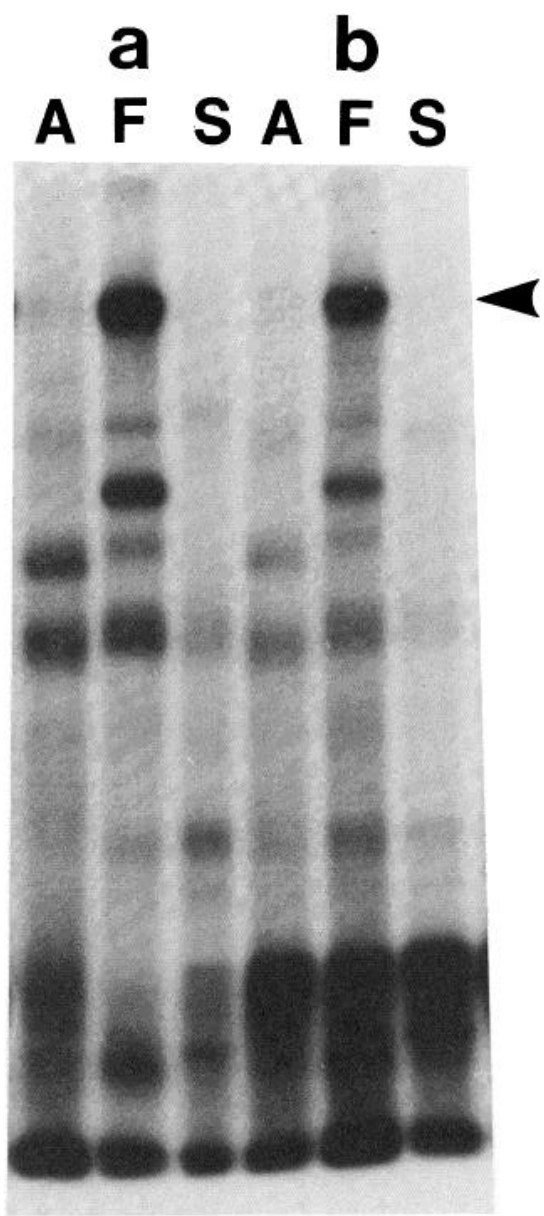

Figure 2. Labeling of cell surface proteins by iodination. Cultures of astrocytes $(A)$, fibroblasts $(F)$, and Schwann cells $(S)$ were labeled with ${ }^{125}$. labeled iodide as described under "Materials and Methods" and then either scraped from the dish $(a)$ or lysed $(b)$. The figure shows analysis of the labeled proteins on a $6 \%$ gel. One can see that by either method of harvesting, the fibroblasts have a distinctive labeled FN band (arrowhead). No such band is found in either astrocytes or Schwann cells in $a$ or $b$.

the cellular form of rat FN (Paul and Hynes, 1984). In the astrocyte immunoprecipitate, however, the smaller band is very faint indeed. It should be noted that 4 times the amount of radioactivity was loaded in the astrocyte lane (Fig. 5, lane 1) than in fibroblast (Fig. 5 , lane 4). Despite this fact, the lower band is barely discernible in the astrocyte FN. As shown in Figure 2, the broad astrocyte FN band is of slightly higher apparent molecular weight than the FN band produced by the fibroblasts. On high resolution gels such as that shown in Figure 5, each form of $\mathrm{FN}$ is resolved into several bands.

Differences produced by post-translational modification. The results described above indicated that astrocytes synthesize and secrete $\mathrm{FN}$ in a form which appears to be slightly larger, both in the dimeric and monomeric forms, than the equivalent protein from fibroblasts. Although this larger size could be the result of a primary protein sequence difference between the two types of FN, it was also possible that it reflected alternative post-translational modifications of the protein. $\mathrm{FN}$ is a glycoprotein known to have asparaginelinked carbohydrate chains, but thought to have no 0 -linked side chains. It has also been shown to be phosphorylated and sulfated (Dunham and Hynes, 1978; Teng and Rifkin, 1979; Ali and Hunter, 1981; Paul and Hynes, 1984). In order to investigate the extent to which post-translational modification might be responsible for the apparent size differences between the fibroblast and astrocyte FNs, 


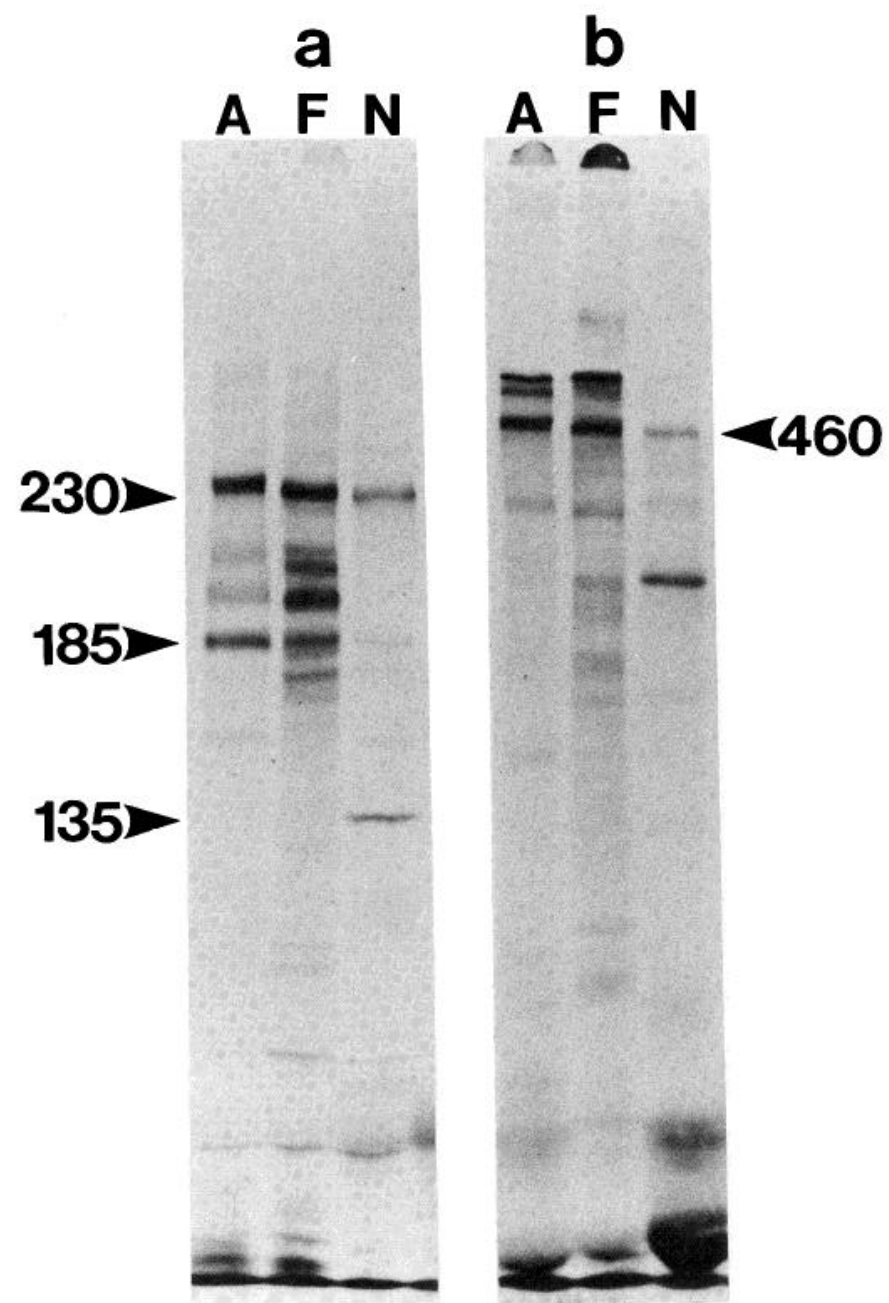

Figure 3. Metabolic labeling of secreted proteins. The culture supernatants from $\left[{ }^{35} \mathrm{~S}\right] \mathrm{methionine-labeled} \mathrm{cultures}$ of astrocytes $(A)$ and fibroblasts $(F)$ were compared with those from the hamster cell line, Nil $8(N)$ by electrophoresis on a long $6 \%$ SDS gel under either reduced (a) or nonreduced $(b)$ conditions. In all three tracks in both $a$ and $b$, one can see the characteristic FN band, but in both cases the astrocyte FN band does not migrate as fast as that from the other two sources. The Nil 8 supernatant was included because, in addition to $\mathrm{FN}$, it provides a number of well characterized bands of known molecular weight for the purpose of comparison. The molecular weight marker bands are (in kilodaltons): 460 , the FN dimer; 230 , the FN monomer; 185 , pro $\mathrm{C} 3$, the precursor form of the $\mathrm{C} 3$ component of complement; 135 , the C3 $\alpha$ chain. (Senger and Hynes, 1978).

\section{cultures of both cell types were metabolically labeled in the presence} of TM.

Figure $5 \mathrm{a}$ shows the results of anti-FN immunoprecipitation of $\left[{ }^{35} \mathrm{~S}\right]$ methionine-labeled supernatants labeled with and without TM treatment. Lanes 1 and 4 show, as already described, the reduced FN from astrocytes and fibroblasts. Lanes 2 and 3 show, respectively, the TM-treated astrocyte and fibroblast forms. In both cases, the FN bands run faster after TM treatment, as one would expect, because of the inhibition of glycosylation (Fig. $5 b$, lanes 1 to 4 ). Differences between FN from the two cell types remain after TM treatment, and the bands are sharper because of the absence of carbohydrate. Both astrocyte and fibroblast FNs resolve into four distinct bands which have been labeled 1, 2, 3, and 4 in Figure 5 , going from heaviest to lightest. This heterogeneity reflects the heterogeneity of the primary sequence among $\mathrm{FN}$ monomers that has been reported previously (Schwarzbauer et al., 1983; Paul and Hynes, 1984). In the case of fibroblasts (Fig. 5a, lane 3), FN band 3 is the most prominent, whereas astrocyte FN (Fig. 5a, lane 2) has

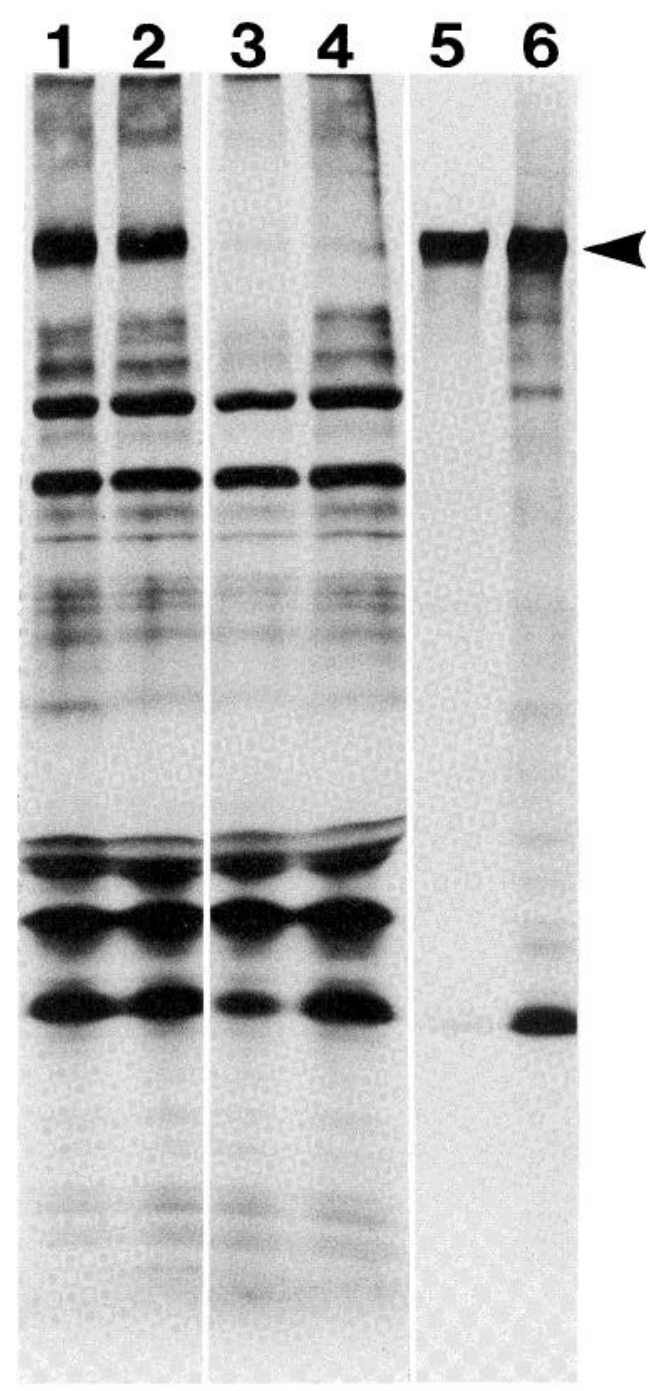

Figure 4. The binding of astrocyte $\mathrm{FN}$ to gelatin and heparin. $\left[{ }^{35} \mathrm{~S}\right]$ Methionine-labeled astrocyte culture supernatant was incubated with either heparin- or gelatin-coupled Sepharose, and the unbound and bound fractions were analyzed on $6 \%$ SDS-PAGE. Track 1 shows the astrocyte supernatant prior to separation. Track 2 shows a control supernatant which has been incubated with uncoupled Sepharose. Tracks 3 and 4 are the supernatants after incubation with gelatin (3)- or heparin-coupled (4) Sepharose. One can see that they are both substantially depleted of the FN band, indicated by the arrowhead. Tracks 5 and 6 are the fractions eluted from the gelatin- and heparin-coupled Sepharose, respectively. Clearly, the prominent band in both cases corresponds to the putative astrocyte FN.

band 2 as its major constituent and only a very faint band 4 is discernible. Therefore, the apparent higher molecular weight of astrocyte $\mathrm{FN}$ reflects a difference in the ratio of $\mathrm{FN}$ subunits between the two cell types. However, there seem to be no bands which are unique to the astrocyte FN. Therefore, even in the absence of glycosylation, fibroblast and astrocyte FNs are distinguishable, showing that the differences between them lie elsewhere.

Sulfation and phosphorylation. When cultures of astrocytes or fibroblasts were labeled with $\left[{ }^{35} \mathrm{~S}_{0} \mathrm{O}_{4}\right.$ or $\left[{ }^{32} \mathrm{P}\right] \mathrm{O}_{4}$, immunoprecipitates of culture supernatants showed labeling of the FN bands (data not shown). The labeling in both cell types appeared equally heavy. In the TM-treated cultures, both the $\left[{ }^{35} \mathrm{~S}^{\mathrm{O}} \mathrm{O}_{4}\right.$ and $\left[{ }^{32} \mathrm{P}\right] \mathrm{O}_{4}$ labels were almost totally absent from any of the four $\mathrm{FN}$ bands produced by either culture (results very similar to Fig. $5 b$, not shown). It is unclear why TM inhibits sulfation and phosphorylation since all the evidence (Teng and Rifkin, 1979; Ali and Hunter, 1981; Paul and Hynes, 1984) shows that, at least for fibroblasts, these modifications occur pre- 


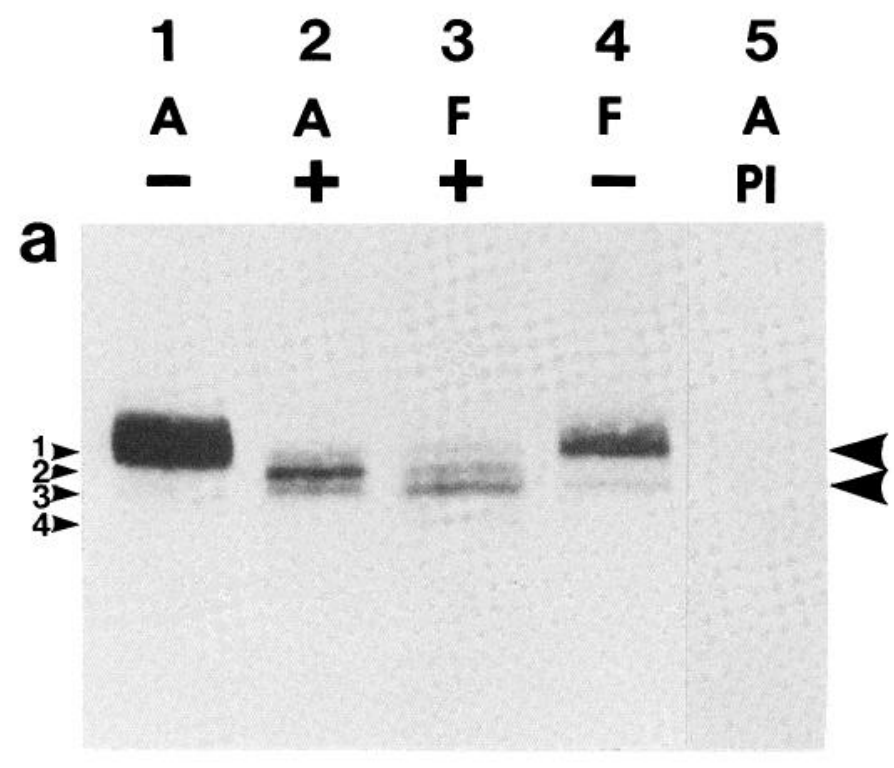

b
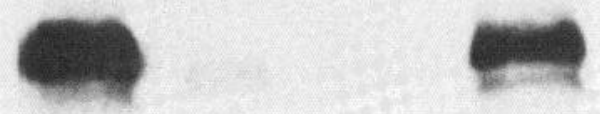

Figure 5. FN subunits in the presence and absence of glycosylation Reduced (5\%) SDS.PAGE analysis of anti-FN immunoprecipitates from $\left[{ }^{35} \mathrm{~S}\right]$ methionine-labeled $(a)$ and $\left[{ }^{3} \mathrm{H}\right]$ glucosamine-labeled $(b)$ culture supernatants from astrocytes $(A)$ and fibroblasts $(F)$, labeled either with $(+$, tracks 2 and 3 ) or without $(-$, tracks 1 and 4 ) TM. In a, one can see that in the absence of TM, both astrocyte and fibroblast FNs resolve into two bands (arrowheads on the right), although the lower one is barely perceptible in the astrocyte case. After TM treatment, both types of FN resolve as four bands, numbered 1 to 4 (arrowheads on the left), but the proportions of the bands are different in the two forms. The gel in $b$ shows that, in the absence of TM, both astrocyte and fibroblast FNs are glycosylated and strongly labeled with $\left[{ }^{3} \mathrm{H}\right]$ glucosamine. The concentration of TM used in these experiments, however, almost completely abolishes this labeling, indicating that the protein is not being glycosylated (tracks 2 and 3). Track 5 in both gels is a control immunoprecipitate of $\left[{ }^{35} \mathrm{~S}\right]$ methionine or $\left[{ }^{3} \mathrm{H}\right]$ glucosamine-labeled astrocyte culture supernatant (no TM treatment) using a preimmune serum.

dominantly on tyrosine and serine, respectively, and not on carbohydrates. It is probable that TM interferes with the enzymatic mechanisms responsible for sulfation and phosphorylation. In any event, the fortuitous inhibition of these reactions by TM shows that the differences between the bands from the TM-treated cultures are not due to differential sulfation or phosphorylation, and it also seems unlikely that such a difference accounts for the difference between the glycosylated forms of the protein.

\section{Discussion}

This study sought to answer the question of whether astrocytes in vitro make $\mathrm{FN}$. The answer is that they synthesize and secrete a protein that can be identified as FN by three different criteria: (1) it has an $M_{r}$ of about 500,000, which becomes 230,000 under reduced conditions (Fig. 3); (2) it binds to heparin and to gelatin (Fig. 4); and (3) it can be specifically precipitated with an antiserum to FN (Fig. 5). This astrocyte FN constitutes a considerable proportion of the total secreted protein of these cells; astrocytes and fibroblasts secrete comparable amounts of FN.

In spite of the large amount of FN produced by cultured astrocytes, the lactoperoxidase surface-labeling experiments (Fig. 2) failed to detect any significant level of FN, suggesting that the FN was not present in significant amounts on the astrocyte cell surface. The immunocytochemical analysis (Fig. 1) largely confirmed this result, except that small amounts of FN were sometimes detected. Our experience was that this was a genuine variation between cultures, and we suggest that this might explain some of the confusion in the literature on whether or not astrocytes have cell surface FN. Although most authors have found astrocytes to be $\mathrm{FN}^{-}$, some have maintained the opposite (see the Introduction). In any event, FN does not seem to be a very good marker for non-neural cells in CNS cultures as, in addition to this variable expression on astrocytes, it rarely confines itself to the borders of a given cell and, frequently, it is difficult to decide in mixed cultures precisely which cells are $\mathrm{FN}^{+}$

In contrast to the results with astrocytes, we found no evidence of either secreted or cell surface FN in Schwann cells. This confirms the results of previous workers who have shown that Schwann cells make laminin but not FN (Cornbrooks et al., 1983).

Although the results presented here demonstrate clearly that astrocytes synthesize and secrete FN, the type of FN they make is not identical with the cellular FN produced by fibroblasts. The differences between astrocyte and fibroblast FN can be summarized as follows.

1. In the normal, glycosylated forms of the protein, astrocyte FN appears to be slightly larger than fibroblast FN, in both reduced and non-reduced states.

2. In the nonglycosylated, nonphosphorylated, nonsulfated form of the protein that results from TM treatment, both astrocyte and fibroblast FNs resolve into four bands, each of which is present in both forms, although the ratios vary between cell types and the lightest band is virtually absent from the astrocyte form (Fig. 5). The quantitative differences were to some extent variable between experiments. On some occasions the predominance of band 2 in astrocytes and band 3 in fibroblasts was less marked. This type of variation is known to occur in cultures of other cell types (Senger et al., 1983). However, the predominance of larger subunits in astrocytes was a consistent finding.

Thus, although one cannot exclude some influence of posttranslational modifications on the size difference observed in the native condition, it seems that the difference is primarily the result of a predominance of larger subunits in astrocyte FN dimers. Furthermore, although there is not an astrocyte-specific subunit, it is probable that the smallest form of the monomer-band 4 - is absent from astrocyte FN. Since it appears as a very faint band in the astrocyte immunoprecipitate, we suspect it is the product of contaminating cells. Although the astrocytes were 95 to $98 \%$ pure, the main contaminants were macrophages and leptomeningeal cells, both of which are known to produce FN (Raff et al., 1979; Alitalo et al., 1980; Van De Water et al., 1981).

The observation that, after TM treatment, FN resolves into a number of discrete spots is not original. Recently, Paul and Hynes (1984) analyzed cellular and plasma FN from both rat and hamster, and showed that after TM treatment the cellular $\mathrm{FN}$ from the rat 1 cell line could be resolved on one-dimensional gels as two bands, the upper of which resolved into four spots and the lower into two spots on two-dimensional electrophoretic analysis. These authors referred to these as $\mathrm{C} 1 \mathrm{a}-\mathrm{d}$, and $\mathrm{C} 2 \mathrm{a}$ and $\mathrm{b}$, respectively. A preliminary comparison of rat 1 cellular FN with that produced by astrocytes and fibroblasts indicated that the middle two bands described here (bands 2 and 3 in Fig. 5) correspond with C1a-b and C1c-d in the rat 1 cellular FN, and our lowest band, band 4, co-migrates with the 
rat $1 \mathrm{C} 2$ spots (data not shown). It seems that the most slowly migrating band in our analysis, band 1 , is not present in rat 1 cellular FN, or in two other established rat cell lines investigated by Paul and Hynes (1984). Thus, it seems that band 1 may represent a subunit that is lost from immortalized rat cell lines.

These results raise questions concerning the molecular basis of these multiple FN subunit proteins. Recent work has shown that, although there is only one FN gene, there are a number of sites within the gene at which exons, or parts of exons, can be omitted or included. Two such sites have been identified so far. Schwarzbauer et al. (1983) showed that, in rat liver, three different FN mRNAs can arise by alternative splicing of the transcript of a single FN gene (Tamkun et al., 1984). Similarly, Kornblihtt et al. (1984) have shown that human FN mRNA can have two forms, one of which has an extra segment incorporated at a site some distance removed from the alternative splice site demonstrated by Schwarzbauer et al. (1983). If these two regions of alternative splicing can be used in any combination (which is not yet clear), they could give rise to six different subunits. However, these two sites are unlikcly to constitutc the entire range possible, as they seem not to allow for as many different forms of the protein as have been discovered. Nor is it clear yet exactly which of the subunits of FN revealed by one- and two-dimensional electrophoretic analysis correspond with which of the alternative mRNAs.

The data presented here also raise a number of questions regarding astrocytes. This study clearly shows that FN is a major secreted protein of astrocytes in culture. However, this FN seems not to be efficiently incorporated into matrix in vitro. One would like to know whether these cells also secrete FN in vivo and what the function of the protein is in the CNS that astrocytes should undertake to make a variant form of the molecule. It seems clear from a number of studies that FN in the adult rat brain is confined to the BMs beneath the pia mater and surrounding blood vessels (Schachner et al., 1978; Kennedy et al., 1980; Paetau et al., 1980a). FN has also been reported to be a constituent of cerebrospinal fluid (Kuusela et al., 1978), although at a concentration so low that plasma contamination has to be considered. It could be that astrocytes contribute to the $\mathrm{FN}$ in one or both of these places. Another possibility is that FN could play a role in developmental events surch as cell migration. It is known to be involved in such phenomena in other developing structures, for example the neural crest, and this hypothesis is particularly altraclive as astrocytes are thought to act as guides for neuroblast migration. In this context, Hatten et al. (1982) described $\mathrm{FN}$ in the path of migrating external granule cells in the developing cerebellum, although this result could not be reproduced either by Schachner et al. (1983) or in this laboratory (R. O. Hynes, R. Patel, and R. H. Miller, al., submitted for publication). Clearly, further research is required to resolve the in vivo role of astrocytes in FN production. From this point of view it is disappointing that there is no FN subunit that is specific to astrocytes and against which specific antisera could have been raised.

\section{References}

Ali. 1. U., and T. Hunter (1981) Structural comparison of fibronectins from normal and transformed cells. J. Biol. Chem. 256: 7671-7677.

Alitalo, K. T. V. Hori, and A. V. Vaheri (1980) Fibronectin is produced by hurnan macrophages. J. Exp. Med. 151:602-613.

Bonner, W. M., and R. A. Laskey (1974) A film detection method for tritiumlabeled protein and nucleic acids in polyacrylamide gels. Eur. J. Biochem. 46: 83-88.

Brockes, J. P., K. L. Fields, and M. C. Raff (1977) A surface antigenic marker for ral Schiwarn cells. Nature 266: 364-366.

Cornbrooks, C. J., D. J. Carey, J. A. McDonald, R. Timpl, and R. P. Bunge (1983) In vivo and in vitro observations on laminin production by Schwann cells. Proc. Natl. Acad. Sci. U. S. A. 80: 3850-3854.

Dunham, J. S., and R. O. Hynes (1978) Differences in the sufated macromolecules synthesized by normal and transformed hamster libroblasts. Biochim. Biophys. Acta 506: 242-255.
Engvall, E., and E. Ruoslahti (1977) Binding of soluble form of fibroblast surface protein, fibronectin, to collagen. Int. J. Cancer 20: 201-205.

Furcht, L. T. (1983) Structure and function of the adhesive glycoprotein fibronectin. Mod. Cell Biol. 1: 53-117.

Hatten, M. E., M. B. Furie, and D. B. Rifkin (1982) Binding of developing mouse cerebellar cells to fibronectin: A possible mechanism for the foundation of the external granular layer. J. Neurosci. 2: 1195-1206.

Hynes, R. O., and A. T. Destree (1978) $10 \mathrm{~nm}$ filaments in normal and transformed cells. Cell 13: 151-163.

Hynes, R. O., and K. M. Yamada (1982) Fibronectins: multifunctional modular glycoproteins. J. Cell Biol. 95: 369-377.

Jones, T. R., E. Ruoslahti, S. C. Schold, and D. D. Bigner (1982) Fibronectin and glial fibrillary acidic protein expression in normal human brain and anaplastic human gliomas. Cancer Res. 42: 168-177.

Kennedy, P. G., R. P. Lisak, and M. C. Raff (1980) Cell type-specific markers for human glial and neuronal cells in culture. Lab. Invest 43: 342-351

Kornblihtt, A. R., K. Vibe-Pedersen, and F. E. Baralle (1984) Human fibronectin: Molecular cloning evidence for two mRNA species differing by an internal segment coding for a structural domain. EMBO J. 3: 221-226.

Kuusela, P., A. Vaheri, J. Palo, and E. Ruoslahti (1978) Demonstration of fibronectin in human cerebrospinal fluid J. Lab. Clin. Med. 92: 595-601.

Laemmli, U. K. (1970) Clearage of structural proteins during the assembly of the head of bacteriophage T4. Nature 227: 680-685.

Liesı, P., D. Dahl, and A. Vaheri (1983) Laminin is produced by early rat astrocytes in primary culture. J. Cell Biol. 96: 920-924.

Mautner, V. M. and R. O. Hynes (1977) Surface distribution of LETS protein in relation to the cytoskeleton of normal and transformed cells. J. Cell Biol. 75: 743-768.

McCarthy, K. D., and J. DeVellis (1980) Preparation of separate astroglial and oligodendroglial cell cultures from rat cerebral tissue. J. Cell Biol. 85: 890-902.

Minier, L. N., R. S. Lasher, and P. F. Erickson (1981) Distribution of the LETS protein (fibronectin) in rat cerebellum. Cell Tissue Res. 214: 491-500.

Paetau, A., K. Mellstrom, A. Vaheri, and M. Haltia (1980a) Distribution of a major connective tissue protein, fibronectin, in normal and neoplastic human nervous tissue. Acta Neuropathol. 51: 17-51.

Paetau, A., K. Mellstrom, B. Westermark, D. Dahl, M. Haltia, and A. Vaheri (1980b) Mutually exclusive expression of fibronectin and glial fibrillary acidic protein in cultured brain cells. Exp. Cell Res. 129: 337-344.

Paul, J. I., and R. O. Hynes (1984) Multiple fibronectin subunits and their posttranslational modifications. J. Biol. Chem. 259: 13477-13487.

Pruss, R. M. (1979) Thy-1 antigen on astrocytes in long-term cultures of rat central nervous system. Nature 280: 688-690.

Raff, M. C., K. L. Fields, S. I. Hakomori, R. Mirsky, R. M. Pruss, and J. Winter (1979) Cell type-specific markers for distinguishing and studying neurons and the major classes of glial cclls in culture. Brain Res. 174: 283-308.

Raff, M. C., R. H. Miller, and M. Noble (1983) A glial progenitor cell that develops in vitro into an astrocyte or an oligodendrocyte depending on culture medium. Nature 303: 390-396.

Rajaraman, R., B. Westermark, A. Vaheri, and J. Ponten (1978) Immunofluorcscence studies on fibronectin distribution patterns during adhesion, deformation, and spreading of human glial and glioma cells. Ann. N. Y. Acad. Sci. 312: 444-449.

Rougon, G., M. Noble, and A. W. Mudge (1983) Neuropeptides modulate the $\beta$-adrenergic response of purified astrocytes in vitro. Nature 305: 715717.

Schachner, M., G. Schoonmaker, and R. O. Hynes (1978) Cellular and subcellular localization of LETS protein in the nervous system. Brain Res. 158: $149-158$.

Schachner, M., A. Faissner, J. Kruse, J. Lindner, D. H. Meier, F. G. Rathjen, and $\mathrm{H}$. Wernecke (1983) Cell type specificity and developmental expression of neural cell-surface components involved in cell interactions and of structurally related molecules. Cold Spring Harbor Symp. Quant. Biol. 48. 557-568.

Schwarzbauer, J. E., J. W. Tamkun, I. R. Lemischka, and R. O. Hynes (1983) Three differerll libronectir mRNAs arise by alternative splicing within the coding region. Cell 35: 421-431. 
Senger, D. R., and R. O. Hynes (1978) C3 component of complement secreted by established cell lines. Cell 15: 375-384.

Senger, D. R., A. T. Destree, and R. O. Hynes (1983) Complex regulation of fibronectin synthesis by cells in culture. Am. J. Physiol. 245: C144-150.

Tamkun, J. W., J. E. Schwarzbauer, and R. O. Hynes (1984) A single rat fibronectin gene generates three different mRNAs by alternative splicing of a complex exon. Proc. Natl. Acad. Sci. U.S.A. 81: 5140-5144.

Teng, M. H., and D. B. Rifkin (1979) Fibronectin from chicken embryo fibroblasts contains covalently bound phosphate. J. Cell Biol. 80: 784791.

Vaheri, A., E. Ruoslànti, B. Westermark, and J. Ponten (1976) A common cell-type specific surface antigen in cultured human glial cells and fibroblasts: Loss in malignant cells. J. Exp. Med. 143: 64-72.

Yamada, K. M. (1983) Cell surface inleractions with extracellular materials. Annu. Rev. Biochem. 52: 761-799. 